\title{
A New Bleaching Protocol for Hyperpigmented Skin Lesions with a High Concentration of All-trans Retinoic Acid Aqueous Gel
}

\author{
Kotaro Yoshimura, M.D., Kiyonori Harii, M.D., Takao Aoyama, Ph.D., Fuminori Shibuya, B.S., and \\ Tatsuji Iga, Ph.D. \\ Tokyo, Japan
}

\begin{abstract}
A new bleaching protocol for skin hyperpigmentation with a higher concentration of all-trans retinoic acid (atRA) aqueous gel than those commercially available is introduced. AtRA aqueous gel $(0.1 \%)$ was applied topically twice a day along with $4 \%$ hydroquinone, $7 \%$ lactic acid ointment to oriental patients with hyperpigmented lesions such as senile lentigines, melasma, and postinflammatory hyperpigmentation. The clinical results of 39 patients treated with $0.1 \%$ atRA aqueous gel were compared to those of 22 patients treated with $0.1 \%$ atRA hydrophilic ointment. Better clinical results and subjective satisfaction were obtained through a significantly shorter period of treatment with $0.1 \%$ atRA aqueous gel than with $0.1 \%$ atRA hydrophilic ointment, although side effects such as erythema and irritation were seen at a higher frequency. It is suggested that our bleaching protocol with a high concentration of atRA aqueous gel in combination with hydroquinone and lactic acid has a strong bleaching ability and a potential as a standard therapy for various kinds of skin lesions with hyperpigmentation.
\end{abstract}

Key words: Retinoic acid-Hydroquinone-BleachingAqueous gel-Hyperpigmentation

All-trans retinoic acid (atRA; tretinoin) has been prescribed as a topical treatment for acne vulgaris for more than 20 years and is also well-known to be effective for certain clinical features of photoaged skin, such as wrinkling and mottled hyperpigmentation (see Refs. 12 and 16 for reviews). Hydroquinone, which acts as an inhibi-

Correspondence to Kotaro Yoshimura, M.D., Department of Plastic and Reconstructive Surgery, University of Tokyo, 7-3-1, Hongo, Bunkyo-Ku, Tokyo, 113 Japan tor of tyrosinase, has been used as a depigmenting agent since its depigmenting ability was first reported in 1936 [17]. Since Kligman et al. [10] reported that the combined use of topical atRA, dexamethasone, and hydroquinone was more effective as a depigmenting formulation than a single use of each reagent, hydroquinone has been widely used in combination with atRA and corticosteroid $[4,18]$. Furthermore, in recent years, hydroquinone has been used in combination with laser therapies $[1,2,8]$, dermabrasion [20], and chemical peelings such as Jessner's solution and glycolic acid $[3,5,13,14]$.

Although Kligman's mixture is well-known and widely used, the bleaching ability is weak and a prolonged period of treatment is required, with variable clinical results. In the present paper, we introduce a new bleaching protocol that employs a high concentration of atRA aqueous gel in combination with hydroquinone and lactic acid. Using this protocol, a remarkable improvement of various skin lesions with hyperpigmentation, such as senile lentigines [22], melasma, and postinflammatory hyperpigmentation, could be obtained with a short period of treatment. Comparison to a protocol with atRA hydrophilic ointment was also made.

\section{Methods}

Combined topical applications of atRA, hydroquinone, and lactic acid were used for skin lesions with hyperpigmentation. Two kinds of $0.1 \%$ atRA ointment were originally prepared at the Department of Pharmacy, University of Tokyo. One was $0.1 \%$ atRA hydrophilic ointment (0.1\% atRA-HO) and the other was $0.1 \%$ atRA aqueous gel (0.1\% atRA-gel). One of the two ointments was topically applied together with 5\% hydroquinone, 7\% lactic acid ointment (HQ-LA ointment), also prepared as described above. Plastibase (petrolatum polyethylene oint- 
Table 1. Summarized data of patients

\begin{tabular}{|c|c|c|c|}
\hline & & $\begin{array}{l}0.1 \% \text { at } \mathrm{RA} \\
\text { hydrophilic ointment }\end{array}$ & $\begin{array}{l}0.1 \% \text { at } \mathrm{RA} \\
\text { aqueous gel }\end{array}$ \\
\hline Total number of patients & & 22 & 39 \\
\hline Scaling & $\begin{array}{l}\text { Number of patients } \\
\text { Treatment time (days) }\end{array}$ & $\begin{array}{l}13 / 22(59 \%) \\
15.6( \pm 7.8)\end{array}$ & $\begin{array}{c}36 / 39(92 \%) \\
5.6( \pm 2.9)^{\mathrm{c}}\end{array}$ \\
\hline Improvement of hyperpigmentation & $\begin{array}{l}\text { Number of patients } \\
\text { Treatment time (days) }\end{array}$ & $\begin{array}{l}18 / 22(82 \%) \\
62.6( \pm 25.0)\end{array}$ & $\begin{array}{l}39 / 39(100 \%) \\
20.8( \pm 12.2)^{\mathrm{c}}\end{array}$ \\
\hline Side effects & $\begin{array}{l}\text { Erythema } \\
\text { Irritation } \\
\text { Erosion }\end{array}$ & $\begin{array}{c}14 / 22(64 \%) \\
10 / 22(45 \%) \\
0 / 22(0 \%)\end{array}$ & $\begin{array}{c}38 / 39(97 \%) \\
24 / 39(62 \%) \\
2 / 39(5 \%)\end{array}$ \\
\hline Clinical result evaluation $(\mathrm{SL} / \mathrm{ML} / \mathrm{PIH})^{\mathrm{b}}$ & $\begin{array}{l}\text { Excellent } \\
\text { Good } \\
\text { Fair } \\
\text { Poor }\end{array}$ & $\begin{array}{l}2(1 / 0 / 1) \\
7(3 / 0 / 4) \\
9(4 / 3 / 2) \\
4(2 / 1 / 1)\end{array}$ & $\begin{array}{c}12(8 / 1 / 3) \\
19(16 / 1 / 2) \\
5(2 / 2 / 1) \\
0(0 / 0 / 0)\end{array}$ \\
\hline
\end{tabular}

${ }^{a}$ Values are mean ( \pm standard deviation).

b UE: upper extremity; LE: lower extremity; SL: senile lentigines; ML: melasma; PIH: postinflammatory hyperpigmentation. Some patients had skin lesions at more than one site of the body.

${ }^{c}$ Significant vs. $0.1 \%$ at RA hydrophilic ointment.

ment base; Taisho Pharmacology, Osaka, Japan) was used as the ointment base of HQ-LA ointment. All three ointments $0.1 \%$ atRA-HO, $0.1 \%$ atRA-gel, and HQ-LA ointment) are pharmacologically unstable, so that fresh ointments were prepared at least once a month. Since 1995, each ointment has been topically applied under signed informed consent to more than 100 oriental patients with hyperpigmented skin lesions such as sinile lentigines, melasma, postinflammatory hyperpigmentation, and nevus spilus.

\section{Treatment Protocol}

One of the two atRA ointments, either $0.1 \%$ atRA-HO or $0.1 \%$ atRA-gel, was applied to the skin lesions twice a day, followed by application of HQ-LA ointment. In the daytime, a broad-spectrum sunscreen cream was concomitantly applied throughout the treatment period. After improvement of hyperpigmentation was obtained, the application of atRA was discontinued, and topical application of corticosteroids $(0.12 \%$ dexamethasone ointment) for 1 to 4 weeks was started to reduce the reactive erythema and inflammation. Throughout the therapy, topical application of HQ-LA ointment was continued except for the cases in which erythema was not reduced after a few weeks' application of corticosteroid and HQLA ointment.

\section{Evaluations of Results}

Clinical Evaluations. The overall clinical results were evaluated at the end of treatment and classified into four grades: "excellent," no clinical pigmentation; "'good," much improved; "fair," moderately improved; and "poor," slight or no improvement. In the cases in which skin lesions in more than one part of the body yielded distinct clinical evaluations, the best evaluation was listed in the table (Table 1).

Colorimetry. In many cases, as an objective measure of the color of the designated lesion and normal skin, a tristimulus colorimeter (Chroma Meter CR-300; Minolta, Osaka, Japan) and a narrow-band spectrophotometer (Mexameter MX 16; COURAGE+KHAZAKA Electric $\mathrm{GmbH}$, Köln, Germany) were used at each clinical visit. $L^{*}, a^{*}$, and $b^{*}$ values were measured with a Minolta CR-300, and melanin and hemoglobin values were measured with a Mexameter. Each spot was measured three times and the average of three measured values was calculated. In these cases, the overall results were objectively evaluated by the melanin values measured with the Mexameter. The difference in the absolute melanin value between a skin lesion and normal skin is referred to as the relative melanin value (RMV) of the skin lesion in this paper, which indicates the intensity of pigmentation relative to the surrounding normal skin. A negative RMV means that the measured spot is lighter than the control. RMV was compared between before and after treatment, and our clinical evaluations were found to correspond to the following classifications of RMV: "excellent," final RMV was 5 or less; "good," final RMV was reduced to less than $20 \%$ of the RMV before treatment; "fair,' final RMV was reduced to less than $60 \%$ of the RMV before treatment; and "poor," final RMV was more than $60 \%$ of the RMV before treatment. The absolute melanin value of normal skin in Japanese and the RMV of hyperpigmented lesions are usually $460-500$ and 20-120, respectively. A RMV of 5 or less is difficult to recognize clinically.

Statistics. The two-sample $t$ test was utilized to compare the treatment periods during which scaling or improvement of hyperpigmentation was achieved. Differences were considered significant for $P$ values $<0.05$. 


\section{Results}

Sixty-one patients with senile lentigines (SL), melasma (ML), and postinflammatory hyperpigmentation (PIH), who were followed up for more than 12 weeks, were analyzed in this study. All of them were oriental: 58 Japanese, 1 Chinese, and 2 Indian. Patients with PIH of less than 12 months' duration were not included. The age of patients varied from 4 to 66 years, and 49 cases were female. The data for the $0.1 \%$ atRA hydrophilic ointment group (HO group) and $0.1 \%$ atRA aqueous gel group (gel group) are summarized in Table 1 . The HO group consisted of 22 patients (6 male and 16 female; age = $42.6 \pm 17.1$, mean \pm SD), and the gel group consisted of 39 patients $(6$ male and 33 female; age $=44.7 \pm 15.3)$. The former included 10 patients with SL (on the face in 8 patients, on the forearms in 5 , and on both in 1), 4 patients with ML (on the face in all patients), and 8 patients with PIH (on the face in 4 patients, on the trunk in 2, and on the upper extremities in 2). The latter included 26 patients with SL (on the face in all patients, of whom 2 also had lesions on the trunk, 8 also on the forearms, and 2 also on the upper extremities), 4 patients with ML (on the face in all patients), and 9 patients (on the face in 4 patients, on the trunk in 2 , on the upper extremities in 2, on the lower extremities in 1). Scaling was seen in 13 cases (59\%) in the HO group after $15.6 \pm$ 7.8 days of treatment, and in 36 cases (92\%) in the gel group. Scaling was observed significantly earlier (at 5.6 \pm 2.9 day) in the gel group than in the HO group. Improvement of hyperpigmentation was obtained in 18 cases (82\%) in the $\mathrm{HO}$ group and in all cases in the gel group. The treatment time required for improvement of the hyperpigmentation, which was equal to the duration of atRA treatment, was $62.6 \pm 25.0$ days in the $\mathrm{HO}$ group and significantly shorter $(19.8 \pm 12.2$ days $)$ in the gel group. As side effects, erythema was seen in 14 cases (64\%) in the HO group and in almost all patients (38 cases; $97 \%$ ) in the gel group. Ten cases $(45 \%)$ in the HO group had irritation of the treated lesions, and there were 24 such cases (62\%) in the gel group. Erosion was seen in two cases in the gel group, and these were thought to have applied $0.1 \%$ atRA-gel more frequently than the proposed protocol. After treatment, in the $\mathrm{HO}$ group, two cases (one case with SL and one with PIH) were evaluated as "excellent," seven cases (three cases with SL and four with PIH) as "good," nine cases (four cases with SL, three with ML, and two with PIH) as "fair," and two cases (two cases with SL, one case with ML, and one with PIH) as " poor." In the gel group, 12 cases ( 8 cases with SL, 1 with ML, and 3 with PIH) were evaluated as "excellent," 19 cases (16 cases with SL, 1 with ML, and 2 with PIH) as "good," 5 cases ( 2 cases with SL, 2 with ML, and 1 with PIH) as "fair," and none as "poor.",

\section{Case Report}

Five representative cases are demonstrated.
Case 1

A 49-year-old woman with sinile lentigines on her left temporal (No. 1) and malar (No. 2) region underwent combined topical applications of $0.1 \%$ atRA gel and HQLA ointment (Fig. 1A; before treatment). On day 3 scaling was seen, and at 2 weeks the hyperpigmentation had disappeared completely, despite the moderate erythema on the treated region. AtRA-gel was discontinued and corticosteroid was topically applied for 2 weeks. Two weeks after the discontinuation of corticosteroid, the erythema disappeared completely, leaving only very slight postinflammatory hyperpigmentation on the temporal lesion (Fig. 1B; after treatment for 6 weeks). HQ-LA ointment was applied throughout the treatment for 6 weeks. The clinical evaluation of this case was "good." The RMVs before treatment were 36.0 (No. 1 ) and 23.3 (No. 2 ), and the final RMVs after 6 weeks of treatment were 6.0 (No. 1) and -2.7 (No. 2), respectively.

\section{Case 2}

A 40-year-old man with a senile lentigine on his right cheek underwent combined topical applications of $0.1 \%$ atRA gel and HQ-LA ointment (Fig. 2A; before treatment). On day 5 scaling was seen, and at 4 weeks atRAgel was discontinued because the hyperpigmentation of the lesion disappeared, leaving only moderate erythema. HQ-LA ointment and corticosteroid were then applied for 3 weeks, but the erythema did not disappear. Therefore, HQ-LA ointment was discontinued and the patient underwent another 2 weeks' application of corticosteroid. Two weeks after discontinuation of corticosteroid, the erythema almost disappeared (Fig. 2B; after 11 weeks' treatment). The clinical evaluation of this case was "good."

\section{Case 3}

A 59-year-old woman had a senile lentigine on her right cheek (Fig. 3A; before treatment). After 2 weeks' treatment with atRA-gel and HQ-LA ointment, the hyperpigmentation disappeared completely, with only mild erythema remaining. AtRA was discontinued and corticosteroid was applied with HQ-LA ointment for 2 weeks. Then, only HQ-LA ointment was applied for another 2 weeks. Hyperpigmentation could be detected neither clinically nor objectively (Fig. 3B; after treatment for 6 weeks). The clinical evaluation of this case was "excellent." The RMV before and after treatment was 33.8 and -1.8 , respectively.

\section{Case 4}

A 32-year-old woman experienced a superficial burn on her left cheek 3 years before her first clinical visit, and postinflammatory hyperpigmentation remained (Fig. 4A; before treatment). On day 5 scaling was seen, and at 2 weeks hyperpigmentation was reduced significantly. The erythema, however, was very mild in this case, so that atRA-gel was applied for another 2 weeks. After atRA was discontinued, corticosteroid and HQ-LA ointment were applied for 2 weeks (Fig. 4B; after treatment for 6 


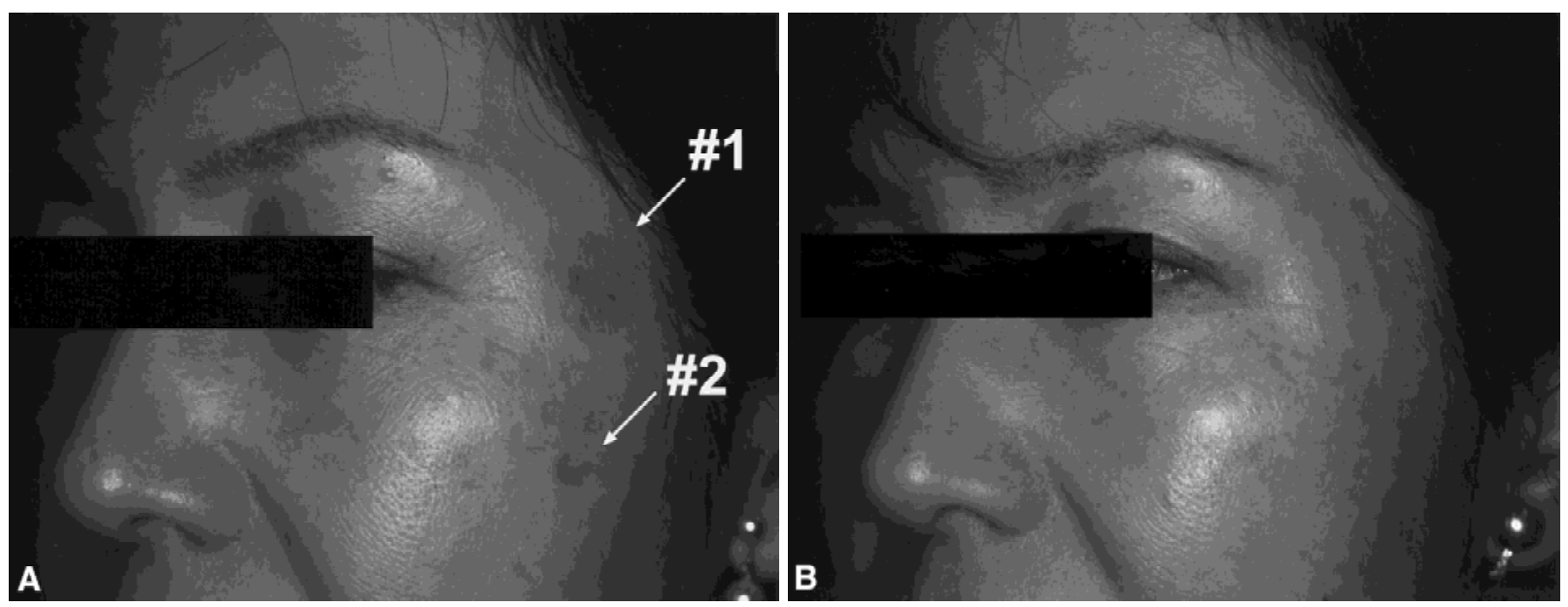

Fig. 1. (A) A pretreatment view of a 49-year-old woman with sinile lentigines on her left temporal (No. 1) and malar (No. 2) region. RMVs were 36.0 (No. 1) and 23.3 (No. 2). (B) After treatment for 6 weeks. RMVs were reduced to 6.0 (No. 1) and -2.7 (No. 2 ), and the clinical evaluation was "good."
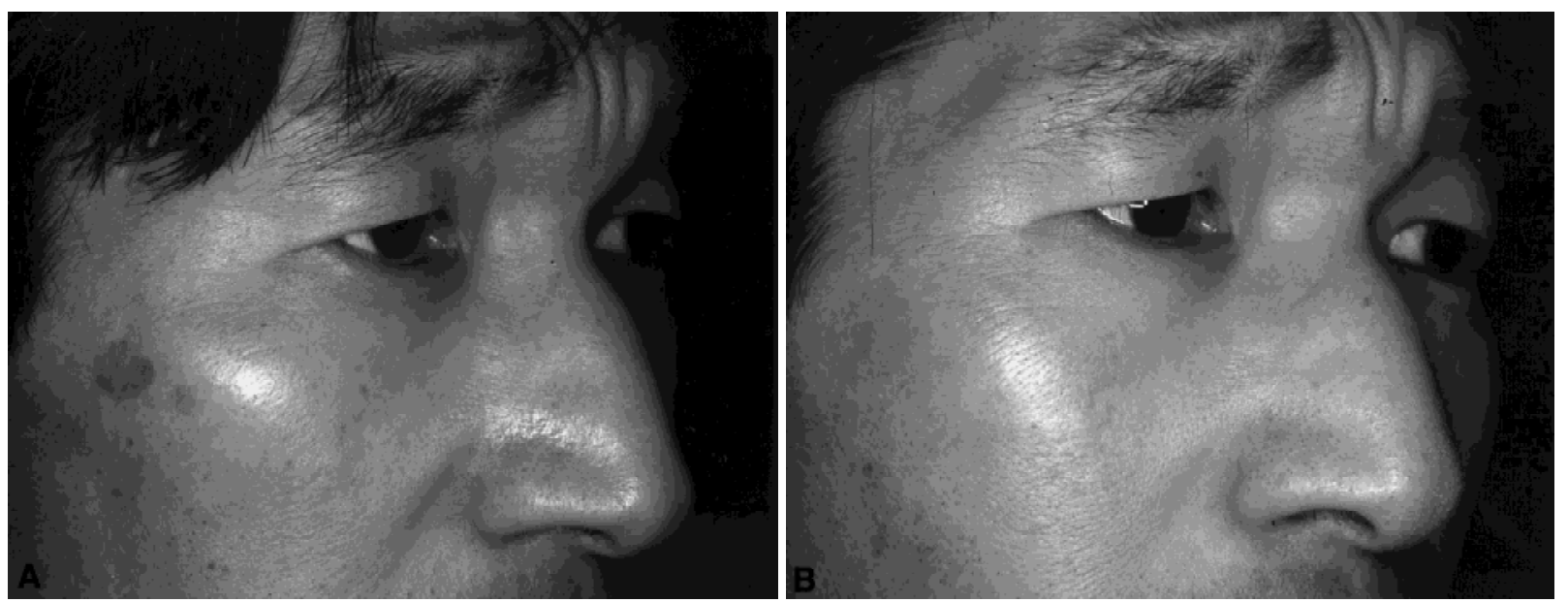

Fig. 2. (A) A pretreatment view of a 40-year-old man with a senile lentigine on his right cheek. (B) After treatment for 11 weeks. The clinical evaluation was "good."
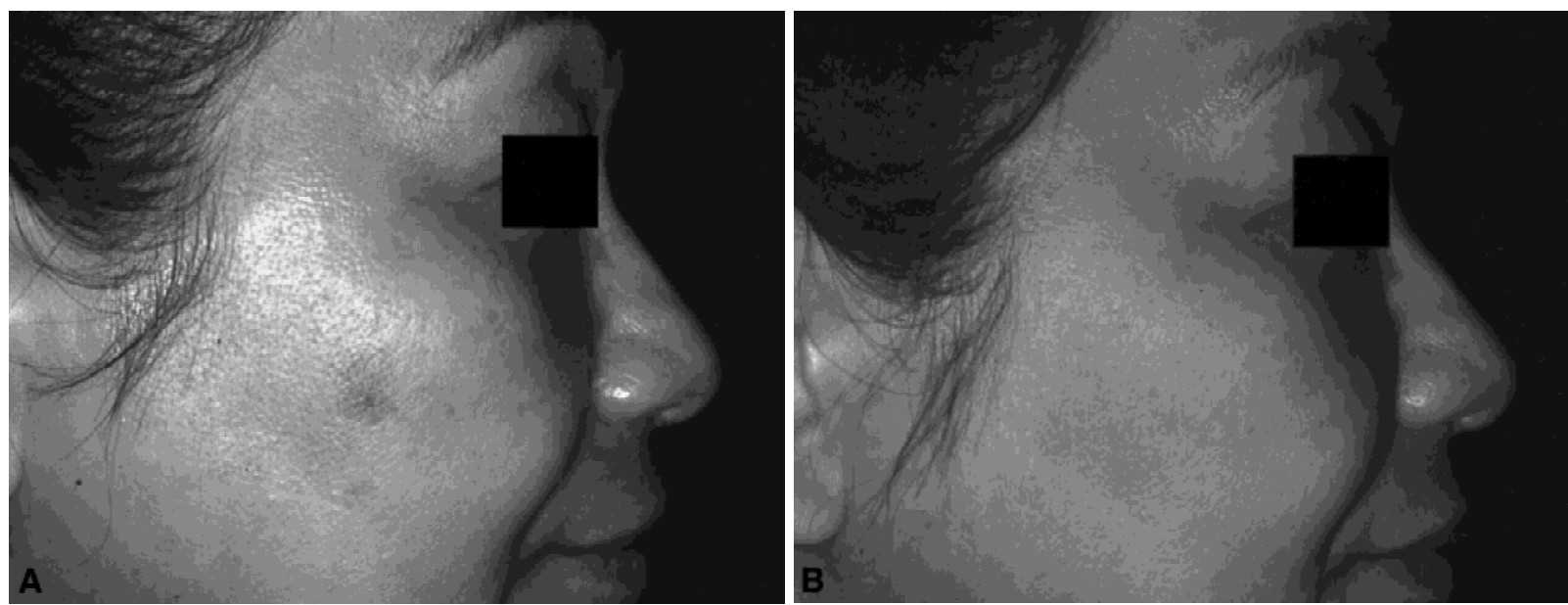

Fig. 3. (A) A pretreatment view of a 59-year-old woman with a senile lentigine on her right cheek. The RMV was 33.8. (B) After 6 weeks of treatment. The RMV was reduced to -1.8 . The clinical evaluation was "excellent." 

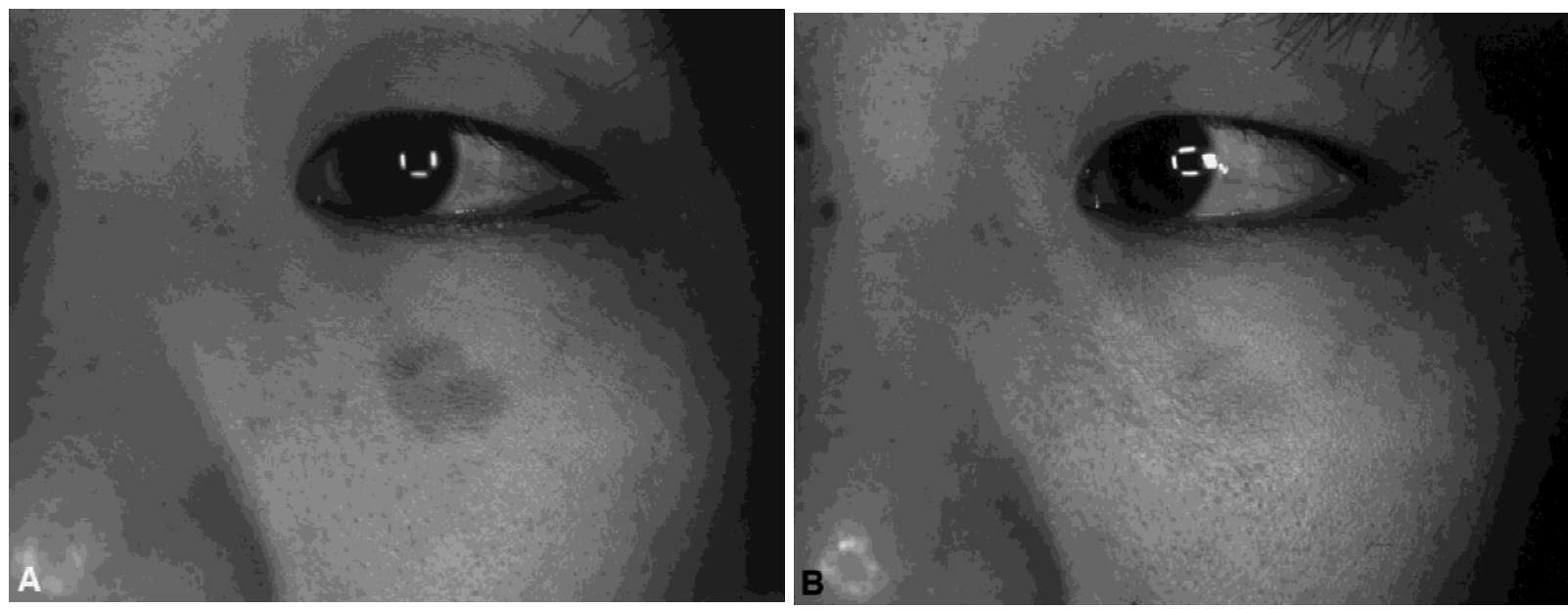

Fig. 4. (A) A pretreatment view of a 32-year-old woman with a postinflammatory hyperpigmentation on her left cheek. The RMV was 41.4. (B) After 8 weeks of treatment. The final RMV was 7.1. The clinical evaluation was "good."
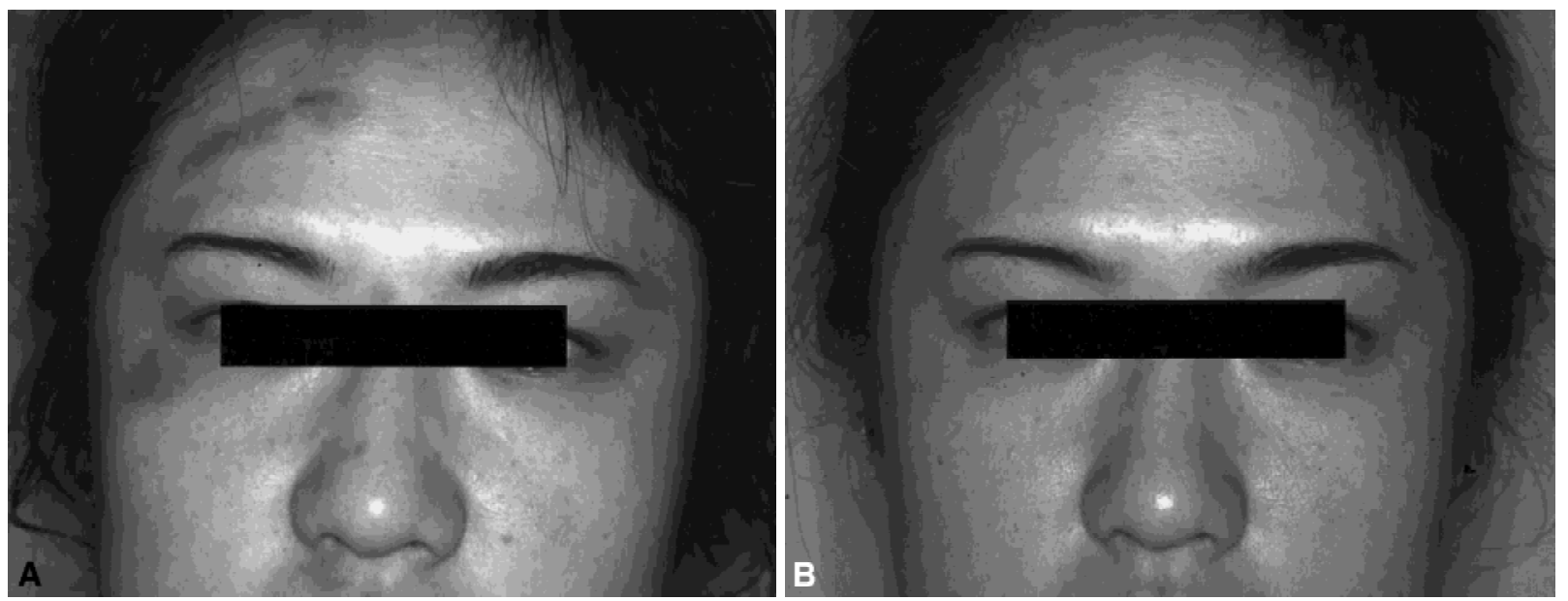

Fig. 5. (A) A pretreatment view of a 28 -year-old woman with postinflammatory hyperpigmentations on face. The RMV was 65.6 on her forehead. (B) After 8 weeks of treatment. The RMV was reduced to 4.0. The clinical evaluation was "excellent."

weeks). The clinical evaluation of this case was "good." The RMV before and after treatment was 41.4 and 7.1 , respectively.

\section{Case 5}

A 28-year-old woman who had suffered multiple superficial abrasive wounds on her face in a traffic accident 1 year before her first clinical visit showed postinflammatory hyperpigmentation at the sites of the wounds (Fig. $5 \mathrm{~A}$; before treatment). Seven days after she started the treatment, scaling was observed, and elimination of the hyperpigmentation was achieved at 6 weeks. She had transient mild erythema during the treatment. Corticosteroid had been used along with HQ-LA ointment for 2 weeks (Fig. 5B; after treatment for 8 weeks). The clinical evaluation was "excellent." The RMV of the pigmentation on the forehead before and after treatment was 65.6 and 4.0, respectively.

\section{Discussion}

We originally prepared atRA ointments in order to evaluate the clinical effectiveness of a more aggressive retinoid treatment as a bleaching protocol than could be performed with commercially available retinoid creams or gels. Hydrophilic ointment and aqueous gel were utilized as the ointment bases of our two original atRA ointments. Our $0.1 \%$ atRA aqueous gel corresponds to a twice higher concentration of atRA than $0.05 \%$ atRA gel (0.05\% Retin-A gel; Ortho Pharmaceutical Corp., Rari$\tan , \mathrm{NJ})$, which is the strongest gel-base atRA ointment commercially available. (Very recently, $0.1 \%$ Retin-A gel also became available for nonfacial use.) Because of differences in the ointment base, much higher absorbance of atRA is assumed to be obtained with $0.1 \%$ atRA aqueous gel than with $0.1 \%$ atRA hydrophilic ointment or with $0.1 \%$ Retin-A cream $[9,21]$. However, since both atRA and hydroquinone are pharmaceutically very un- 
stable, our ointments were prepared at least once a month, stored in a dark and cool place $\left(4^{\circ} \mathrm{C}\right)$, and never used more than 1 month after the preparation.

In the gel group, improvement of the hyperpigmentation was obtained in all cases with only $20.8 \pm 12.2$ days of treatment, much shorter than the $62.6 \pm 25.0$ days in the HO group. Furthermore, 31 of 39 cases (79\%) were clinically evaluated as "excellent" or "good" in the gel group, as opposed to only 9 of $22(41 \%)$ cases in the HO group. There have been a number of reports on the clinical effectiveness of single-use treatment with topical atRA for hyperpigmented lesions $[6,11,19]$. However, it usually took as many as 300 days to get satisfactory results with a lower success rate than our protocol $[6,11,19]$.

After a preliminary trial with a small number of cases, Klingman et al. [10] reported that increasing the concentration of tretinoin to $0.2 \%$ accentuated irritation without a corresponding gain in effectiveness. In a recent study, Griffiths et al. [7] also claimed that atRA at 0.1 and $0.025 \%$ produced similar clinical and histologic changes in patients with photoaging, despite the significantly greater incidence of irritation with the higher concentration. However, in our bleaching protocol combined with hydroquinone and lactic acid, atRA aqueous gel led to much better objective and clinical results and subjective satisfaction than atRA hydrophilic ointment. The much more effective delivery of atRA to the skin by the gel base enabled a greater improvement and shorter treatment period than could be obtained by hydrophilic ointment, although side effects such as erythema and irritation were seen more frequently in the gel group as had been expected. This protocol may not be recommended for people expecting merely cosmetic improvement. However, this protocol shows great promise as a medical therapy for various pigmented skin lesions. Indeed, only a few of more than 100 patients dropped out from this therapy because of the side effects.

The importance of the absorption of atRA was also suggested by the distinct reactions in different parts of the body [19]. Although most of our cases had the skin lesion on the face, the reaction to the treatment was found to be milder and slower on the trunk and extremities, especially on the lower extremities. Differences in the permeability and vascularity of the skin, associated with the absorption ability and wound healing, are suspected to be the main reasons responsible for the differences in skin reaction [15]. Therefore, at present, we are using $0.2 \%$ atRA-gel for the trunk and upper extremities and $0.4 \%$ atRA-gel for the lower extremities, with much better clinical results than with $0.1 \%$ atRA-gel. Satisfactory clinical results with our latest protocol were achieved also for lesions of the extremities and trunk and nevus spilus.

In some cases, very light pigmentation was observed after treatment on exactly the same area as that on which the ointments were applied. This may suggest that inflammatory hyperpigmentation was induced by this treatment. Moreover, through an objective examination with the Mexameter, this small rebound of RMV could be detected after the cessation of atRA in some cases. In many cases, the RMV at the time of cessation of atRA was negative, yet the final RMV in some of these was elevated to $0-10$. Although the final RMV remained negative in some cases, the depigmentation was usually clinically undetectable, and no patients have complained about it.

In order to reduce undesirable side effects such as erythema and irritation and with the expectation of a synergistic depigmenting effect, topical application of corticosteroid has been recommended and widely performed $[4,10]$. However, Pathak et al. [18] indicated that corticosteroids are not essential in depigmenting formulations. Based on the results of our preliminary trials, the beneficial effect of atRA is considerably reduced by corticosteroids, which is known to show adverse effects of retinoids to keratinocytes and fibroblasts. Accordingly, we believe that corticosteroids should not be used concomitantly with atRA. In our protocol, a corticosteroid ointment was always used after discontinuation of atRA as short as possible to reduce erythema. In conclusion, the unique points of the present protocol are as follows: (a) our protocol utilizes an aqueous gel base with a high concentration of atRA, and (b) we never use corticosteroids concomitantly with atRA. Although our protocol is a two-step precedure (bleaching and reducing erythema), unlike Kligman's mixture, the above two points are critical to obtain strong bleaching effects in a short period of treatment.

\section{Conclusion}

Our bleaching protocol for various skin lesions with hyperpigmentation is introduced. Two kinds of $0.1 \%$ atRA ointments (aqueous gel and hydrophilic ointment) were originally prepared and either was topically applied with $5 \%$ hydroquinone and $7 \%$ lactic acid. Much better clinical results and subjective satisfaction were obtained in a significantly shorter period of treatment with $0.1 \%$ atRA aqueous gel than with $0.1 \%$ atRA hydrophilic ointment, despite the higher frequency of side effects. It is concluded that a high concentration atRA aqueous gel has a potential as a standard bleaching therapy for various kinds of skin lesions with hyperpigmentation.

\section{References}

1. Apfelberg DB: Ultrapulse carbon dioxide laser with $\mathrm{CPG}$ scanner for full-face resurfacing for rhytids, photoaging, and acne scars. Plast Reconstr Surg 99:1817, 1997

2. Bekhor PS: The role of pulsed laser in the management of cosmetically significant pigmented lesions. Australas J Dermatol 36:221, 1995

3. Burns RL, Prevost-Blank PL, Lawry MA, Lawry TB, Faria DT, Fivenson DP: Glycolic acid peels for postinflammatory hyperpigmentation in black patients. A comparative study. Dermatol Surg 23:171, 1997

4. Gano SE, Garcia RL: Topical tretinoin, hydroquinone, and 
betamethasone valerate in the therapy of melasma. Cutis 23:239, 1979

5. Garcia A, Fulton JE: The combination of glycolic acid and hydroquinone or kojic acid for the treatment of melasma and related conditions. Dermatol Surg 22:443, 1996

6. Griffiths CEM, Goldfarb MT, Finkel LJ, Roulia V, Bonawitz M, Hamilton TA, Ellis CN, Voorhees JJ: Topical tretinoin (retinoic acid) treatment of hyperpigmented lesions associated with photoaging in Chinese and Japanese patients: A vehicle-controlled trial. J Am Acad Dermatol 30:76, 1994

7. Griffiths CEM, Kang S, Ellis CN, Kim KJ, Finkel LJ, Ortiz-Ferrer LC, White GM, Hamilton TA, Voorhees JJ: Two concentrations of topical tretinoin (retinoic acid) cause similar improvement of photoaging but different degrees of irritation. Arch Dermatol 131:1037, 1995

8. Ho C, Nguyen Q, Lowe NJ, Griffin ME, Lask G: Laser resurfacing in pigmented skin. Dermatol Surg 21:1035, 1995

9. Kammerau B, Klebe U, Zesch A, Schaefer H: Penetration, permeation, and resorption of 8-methoxypsoralen. Arch Dermatol Res 255:31, 1976

10. Kligman AM, Willis I: A new formula for depigmenting human skin. Arch Dermatol 111:40, 1975

11. Kimbrough-Green CK, Griffiths CEM, Finkel LJ, Hamilton TA, Bulengo-Ransby SM, Ellis CN, Voorhees JJ: Topical retinoic acid (tretinoin) for melasma in black patients. Arch Dermatol 130:727, 1994

12. Kligman AM, Grove GL, Hirose R, Leyden JJ: Topical tretinoin for photoaged skin. J Am Acad Dermatol 15:836, 1986

13. Lawrence N, Cox SE, Brody HJ: Treatment of melasma with Jessner's solution versus glycolic acid: A comparison of clinical efficacy and evaluation of the predictive ability of Wood's light examination. J Am Acad Dermatol 36:589, 1997

14. Lim JT, Tham SN: Glycolic acid peels in the treatment of melasma among Asian women. Dermatol Surg 23:177, 1997

15. McKenzie AW, Stoughton RB: Method for comparing percutaneous absorption of steroids. Arch Dermatol 86:608, 1962

16. Noble S, Wagstaff AJ: Tretinoin-A review of its pharmacological properties and clinical efficacy in the topical treatment of photodamaged skin. Drugs Aging 6:479, 1995

17. Oettel H: Die Hydrochinonenvergiftung. Arch Exp Path Pharmacol 183:319, 1936

18. Pathak MA, Fitzpatrick TB, Kraus EW: Usefulness of retinoic acid in the treatment of melasma. J Am Acad Dermatol 15:894, 1986

19. Rafal ES, Griffiths CEM, Ditre CM, Finkel LJ, Hamilton TA, Ellis CN, Voorhees JJ: Topical tretinoin (retinoic acid) treatment for liver spots associated with photodamage. $\mathrm{N}$ Engl J Med 326:368, 1992

20. Tezuka T, Saheki M, Kusuda S, Umemoto K: Treatment of nonhairy melanocytic macules by dermabrasion and topical application of 5\% hydroquinone monobenzyl either cream. J Am Acad Dermatol 28:771, 1993

21. Treffel P, Gabard B: Skin penetration and sun protection factor of ultra-violet filters from two vehicles. Pharm Res 13:770, 1996

22. Yoshimura K, Harii K: A new treatment for senile lentigines. J Jpn Plast Reconstr Surg 17:630, 1997 